\title{
Superpixel Classification Based Optic Cup Segmentation
}

\author{
Jun Cheng ${ }^{1, \star}$, Jiang Liu ${ }^{1}$, Dacheng Tao ${ }^{2}$, Fengshou Yin ${ }^{1}$, \\ Damon Wing Kee Wong ${ }^{1}$, Yanwu $\mathrm{Xu}^{1}$, and Tien Yin Wong ${ }^{3,4}$ \\ 1 Institute for Infocomm Research, Agency for Science, \\ Technology and Research, Singapore \\ 2 University of Technology, Sydney, Australia \\ dacheng.tao@uts.edu.au \\ 3 Singapore Eye Research Institute, Singapore \\ 4 National University of Singapore, Singapore \\ ophwty@nus.edu.sg
}

\begin{abstract}
In this paper, we propose a superpixel classification based optic cup segmentation for glaucoma detection. In the proposed method, each optic disc image is first over-segmented into superpixels. Then mean intensities, center surround statistics and the location features are extracted from each superpixel to classify it as cup or non-cup. The proposed method has been evaluated in one database of 650 images with manual optic cup boundaries marked by trained professionals and one database of 1676 images with diagnostic outcome. Experimental results show average overlapping error around $26.0 \%$ compared with manual cup region and area under curve of the receiver operating characteristic curve in glaucoma detection at 0.811 and 0.813 in the two databases, much better than other methods. The method could be used for glaucoma screening.
\end{abstract}

\section{Introduction}

Glaucoma is a chronic eye disease in which the optic nerve is progressively damaged. It is the second leading cause of blindness, and is predicted to affect around 80 million people by 2020 [1. Although glaucoma cannot be cured, its progression can be slowed down by treatment. Therefore, it is important to detect it in time. The cup-to-disc ratio (CDR) [2] is a widely used indicator for glaucoma. A larger CDR indicates a higher risk of the disease. In current clinical practice, the $\mathrm{CDR}$ is measured manually. However, manual measurement is subjective, time consuming and expensive for large scale screening. Recently, automated CDR measurement from $2 \mathrm{D}$ fundus images starts to receive some attention. In 2D images, the optic disc can be divided into two distinct zones, namely, a central bright zone called the optic cup (in short, cup) and a peripheral region called

* This work is funded by Singapore A*STAR SERC Grant (092-148-0073). 
the neuroretinal rim (in short, rim), as shown in Fig. 1. The CDR is computed as the ratio of the vertical cup diameter to the vertical disc diameter clinically. Accurate segmentation of the cup is essential for CDR measurement. In this paper, we focus on automatic cup segmentation only.

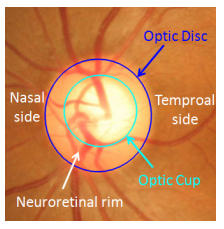

Fig. 1. Major structures of the optic disc

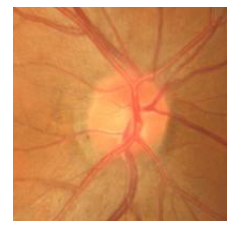

Fig. 2. An example with no pallor

Cup segmentation in 2D fundus images is a challenging task as $2 \mathrm{D}$ images do not carry depth information, which is the primary indicator of the cup boundary. In these images, one landmark for cup detection is the area of maximum color contrast inside the optic disc, i.e., pallor. Another landmark is the vessel bends at the cup boundary. Several methods have been proposed for cup segmentation from 2D images. In [3], thresholding is used. It is essentially based on pallor information. However, in many subjects from screening, there is no obvious pallor to mark the cup boundary. Fig. 2] shows such an example. In 4], 5], vessel bends are used. The challenge is to correctly identify vessel bends. Xu et al. proposed a sliding window and regression based method [6. However, the sliding window strategy requires heavy computational cost. Recently, Yin et al. [7] developed a deformable model based approach, where the initialization of the cup boundary is based on pallor combined with prior knowledge of cup.

One main challenge in cup segmentation is to determine the cup boundary in images with weak or no pallor. In such scenarios, it is difficult to determine the cup based on intensity only. Although vessel bends are potential landmarks, they suffer from high false alarm. In addition, these methods often require a set of heuristic parameters which raises the concern of the robustness of the methods. In this paper, we propose superpixel classification for cup segmentation. The proposed method embeds the prior knowledge into superpixel classification by using location feature to overcome the absence of pallor. In addition, other challenges such as uneven illuminations within and between images have also been newly discussed and explored. Contrast enhanced intensities, mean removed intensities and center surround statistics are proposed to overcome these challenges accordingly. Based on the detected cup, CDR is computed for glaucoma detection.

The rest of paper is organized as follows. In Section 2, we introduce superpixel classification based cup segmentation including the generation of superpixels, the extraction of feature from superpixels for the classification and the classification of superpixels for cup estimation. Section 3 shows the experimental results including the accuracy for cup segmentation, the CDR and the glaucoma detection. Discussions and conclusions are presented in the last section. 


\section{Methodology}

The proposed superpixel classification based method comprises: an oversegmentation step to divide the disc image into superpixels; a feature extraction step to compute features from each superpixel; a classification step to determine each superpixel as cup or non-cup (rim) and to estimate the boundary. Fig. 3 shows the procedure. Superpixel is an image patch which is better aligned with intensity edges than a rectangular patch. It captures redundancy in the image and reduces the complexity of subsequent processing. This paper uses the simple linear iterative clustering [8] algorithm (SLIC) to aggregate nearby pixels into superpixels in retinal fundus images. Compared with other superpixel methods, SLIC is fast, memory efficient and has excellent boundary adherence. It is also simple to use with only one parameter, i.e., the number of desired superpixels $k$. Next, features from superpixels are extracted to classify them as cup or non-cup.

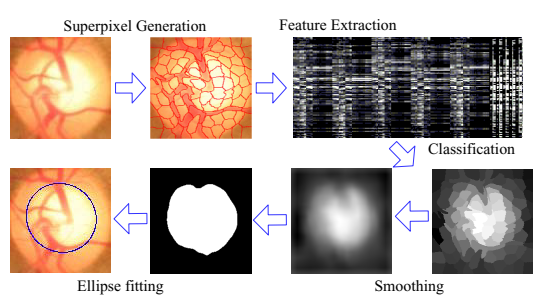

Fig. 3. Superpixel based optic cup segmentation

\subsection{Feature Extraction}

Many features such as color, appearance, gist, location and texture can be extracted for superpixel classification. To compute good features for cup segmentation, it is important to understand the main challenges in the task. The first challenge is that the contrast between rim and cup varies largely from one image to another. Therefore, it is beneficial to enhance the image contrast. In this paper, we apply histogram equalization on the red $r$, green $g$, and blue $b$ channels to get three contrast enhanced intensity maps $H E(r), H E(g), H E(b)$, where $H E(\cdot)$ denotes the function of histogram equalization. The second challenge is the illumination changes from one image to another, i.e., some images look darker and some brighter. To overcome this issue, the mean removed intensity maps are also computed as $r-\bar{r}, g-\bar{g}, b-\bar{b}$, where $\bar{x}$ denotes the mean value in channel $x$. Combining them with the contrast enhanced intensity maps, we obtain six intensity based maps $I=\left[I_{1}, \cdots, I_{6}\right]$. The first type of features from a superpixel are the mean intensities $M I$ of pixels within the superpixel. Mathmatically, $M I$ for $j^{\text {th }}$ superpixel $S P_{j}$ with $n_{j}$ pixels is computed as: $M I(i)=\frac{1}{n_{j}} \sum_{(x, y) \in S P_{j}} I_{i}(x, y)$, for $i=1, \cdots, 6$. 
Unbalanced illumination across the optic disc within the image is another challenge. For example, the temporal side of the disc is often brighter than the nasal side. In this paper, we compute center surround statistics (CSS) to overcome this issue. CSS are useful as they are computed from the difference between image maps, which are less sensitive to unbalanced illumination across the disc. To compute CSS, nine spatial scale dyadic Gaussian pyramids are generated with a ratio from 1:1 (level 0) to 1:256 (level 8). Then center-surround operation between center finer levels $c=2,3,4$ and surround coarser levels $s=c+d$, with $d=3,4$ is applied to obtain six maps computed at levels of 2-5, 2$6,3-6,3-7,4-7$, and 4-8 from an image channel [9]. Because of the scale difference, surround levels are interpolated to be the same size as the corresponding center levels, and then they are subtracted to generate the center surround difference maps. In this paper, we compute the maps from $r, g$ and $b$ channels to get $6 \times 3=18$ maps. The CSS features are then computed as the mean and variance of these maps within superpixels. Denoting $M_{i}, i=1,2, \cdots, 18$, as the $i^{\text {th }}$ map, the feature $C S S$ consists of the mean $M_{\mu}$ and variance $M_{\sigma}$ of maps within the superpixels, i.e., $C S S=\left[M_{\mu} M_{\sigma}\right]$, where $M_{\mu}$ and $M_{\sigma}$ are computed as follows:

$$
M_{\mu}(i)=\frac{1}{n_{j}} \sum_{(x, y) \in S P_{j}} M_{i}(x, y), \quad M_{\sigma}(i)=\frac{1}{n_{j}} \sum_{(x, y) \in S P_{j}}\left(M_{i}(x, y)-M_{\mu}(i)\right)^{2}
$$

Combining $C S S$ with $M I$ yield a feature with $18 \times 2+6=42$ dimensions. Besides the above, blood vessels from the cup and rim often look quite similar. In order to differentiate the vessels from the two regions, $M I$ and $C S S$ from neighboring superpixels are also included as context features in the classification of current superpixel. By choosing proper desired number of superpixels, a superpixel from vessels often have two or more non-vessel neighbors. Therefore, the vessels from the cup and rim can be better differentiated from their neighbors. In this paper, four neighboring superpixels for current superpixel $S P_{j}$ are obtained and denoted as $S P_{j_{1}}, S P_{j_{2}}, S P_{j_{3}}$ and $S P_{j_{4}} . S P_{j_{1}}$ is determined as the first superpixel by moving out of the current superpixel horizontally to the left from its center. Similarly, $S P_{j_{2}}, S P_{j_{3}}$ and $S P_{j_{4}}$ are determined by moving right, up and down.

Since intensities and CSS features rely on color changes from cup to rim, they do not work well for images with very weak or no color changes from the cup to rim. To obtain a reliable result in such a scenario, we make use of the prior knowledge that the cup is usually the center part of the disc. For this purpose, we further include the distance $D$ between the center of superpixel $S P_{j}$ and the center of optic disc as location information. Mathematically, $D$ is computed as $D=\sqrt{\left(\frac{x_{c}-x_{j}}{h}\right)^{2}+\left(\frac{y_{c}-y_{j}}{w}\right)^{2}}$, where $\left(x_{c}, y_{c}\right)$ denotes the coordinate of the disc centre, $\left(x_{j}, y_{j}\right)$ denotes the coordinate of the centre of $S P_{j}, h$ and $w$ denotes the height and width of the disc, respectively. By including the location information, the prior knowledge would be automatically embedded into classification. The final feature has $42 \times 5+1=211$ dimensions. 


\subsection{Superpixel Classification}

The support vector machine (SVM) is used for the two-class classification in our application. The LIBSVM [10] with linear kernel is used in our experiments as we find that the nonlinear mapping using the radial basis function kernel does not improve much the performance. In the training step, we randomly obtain the same number of superpixels from the cup and the rim. Two-fold crossvalidation is adopted to determine the SVM parameters. In the testing, instead of directly using the binary classification results from LIBSVM, we obtain the output values from the SVM decision function and smooth them with a mean filter, as illustrated in Fig. 3. The smoothed decision values are then used to get the binary decisions for all pixels by the default threshold used in SVM. The largest connected object is obtained and its boundary is used as the raw estimation. The best fitted ellipse [11 is computed as the cup boundary. The ellipse fitting here is beneficial to overcome the noise introduced by vessels at the inferior and superior sector of the cup.

\section{Experimental Results}

Two data sets are used. The first set includes 650 different eye images from Singapore Malay Eye Study (SiMES). Manual disc and cup boundaries are available for the 650 images. The 650 images are randomly divided into 325 images for training and 325 images for testing. The second set includes 1676 images of a population based data from Singapore Chinese Eye Study (SCES). Only diagnostic outcome is available for SCES. All SCES data are used in evaluating the performance of glaucoma screening using the proposed method. The disc boundaries are obtained following the method in [12. Among the 2326 eyes, 168 of SiMES and 46 of SCES eyes are diagnosed as glaucomatous by ophthalmologists. The overlapping error $E$ is computed as one evaluation metric to examine the difference between automated and manual cup: $E=1-\frac{\operatorname{Area}(S \cap M)}{\operatorname{Area}(S \cup M)}$, where $S$ and $M$

Table 1. Mean overlapping error $\mu_{E}$ at different parameters

\begin{tabular}{c|c|c|c|c|c|c}
\hline & no filter & $5 \times 5$ & $10 \times 10$ & $15 \times 15$ & $20 \times 20$ & $25 \times 25$ \\
\hline \hline 50 & $29.3 \%$ & $28.4 \%$ & $27.7 \%$ & $27.2 \%$ & $26.8 \%$ & $26.6 \%$ \\
\hline 100 & $27.1 \%$ & $26.5 \%$ & $26.3 \%$ & $26.1 \%$ & $26.0 \%$ & $26.1 \%$ \\
\hline 200 & $26.6 \%$ & $26.2 \%$ & $25.9 \%$ & $25.9 \%$ & $25.8 \%$ & $25.8 \%$ \\
\hline 400 & $26.6 \%$ & $26.1 \%$ & $26.0 \%$ & $25.9 \%$ & $25.8 \%$ & $25.9 \%$ \\
\hline
\end{tabular}

Table 2. Performance by various methods

\begin{tabular}{c|c|c|c|c|c}
\hline Measurement & $\mu_{E}$ & \multicolumn{2}{|c|}{ Mean CDR error $\mu_{\delta}$} & \multicolumn{2}{c}{ AUC } \\
\hline \multirow{2}{*}{ Data set } & \multirow{2}{*}{ SiMES } & \multicolumn{2}{|c|}{ SiMES } & \multirow{2}{*}{ SiMES } & \multirow{2}{*}{ SCES } \\
\cline { 3 - 5 } & & glaucoma & healthy & & \\
\hline Threshold 3 & $53.5 \%$ & 0.141 & 0.129 & 0.638 & 0.574 \\
\hline ASM 7 & $31.3 \%$ & 0.121 & 0.088 & 0.716 & 0.756 \\
\hline Regression 6 & $28.4 \%$ & 0.133 & 0.091 & 0.729 & 0.767 \\
\hline$M I+C S S$ & $28.3 \%$ & 0.108 & 0.084 & 0.734 & 0.694 \\
\hline$M I+D$ & $27.0 \%$ & 0.074 & 0.102 & 0.757 & 0.768 \\
\hline$C S S+D$ & $26.8 \%$ & 0.102 & 0.078 & 0.782 & 0.789 \\
\hline$M I+C S S+D$ (Proposed) & $26.0 \%$ & 0.100 & 0.075 & 0.811 & 0.813 \\
\hline Manual & - & - & - & 0.839 & - \\
\hline & & & & &
\end{tabular}




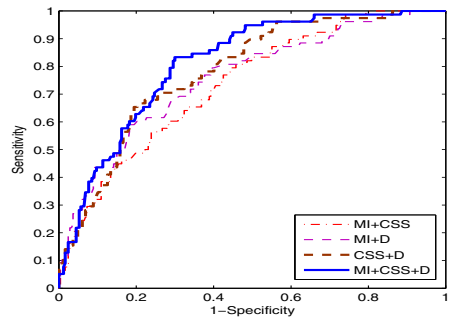

(a) ROC using different features in SiMES

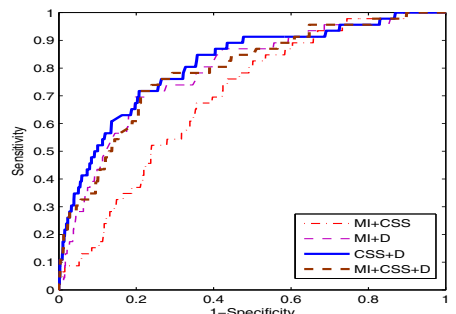

(c) ROC using different features in SCES

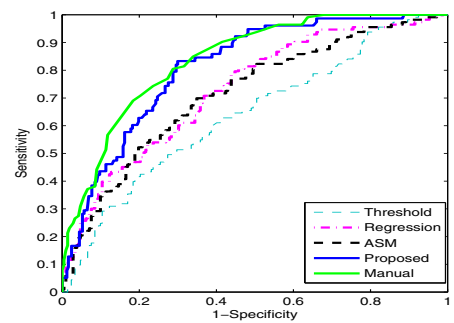

(b) ROC using different methods in SiMES

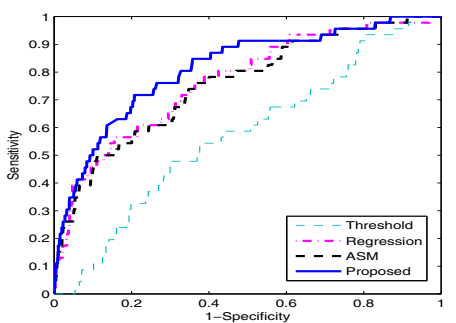

(d) ROC using different methods in SCES

Fig. 4. ROC of glaucoma detection

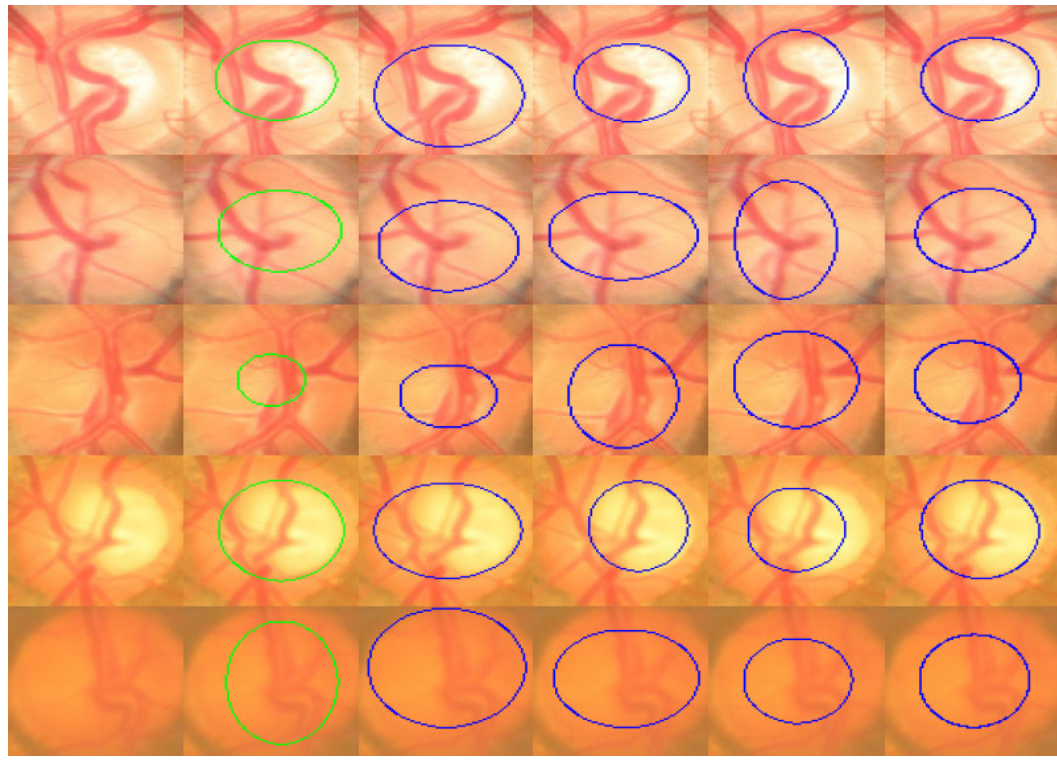

(a) Original (b) Manual (c) Threshold (d) ASM (e) Regression (f) Proposed

Fig. 5. Sample results. From top to bottom, the manual CDR for the five examples from top to bottom are: $0.54,0.55,0.35,0.68$ and 0.83 . The CDR by the proposed method are $0.55,0.55,0.54,0.65$, and 0.63 respectively. 
denote the segmented and the manual cup respectively. In addition, the CDR accuracy is also evaluated using CDR error, computed as $\delta=\left|C D R_{m}-C D R\right|$, where $C D R_{m}$ denotes the manual CDR. The glaucoma detection accuracy is evaluated using the area under curve (AUC) of the receiver operating characteristic (ROC) curve.

The first set of experiments was carried out to evaluate the performance under different parameters. Table 1 shows the mean overlapping error $\mu_{E}$ for the number of superpixels $k$ at 50,100, 200 and 400 in combined with different filter sizes from $5 \times 5$ to $25 \times 25$ as well as the case without a filter. The results show $k=50$ is too small for this application while there is mild improvement when $k$ increases from 100 to 400 . It is shown that the results with a filter are better than that without. The size of filter only affects the performance very slightly from $15 \times 15$ to $25 \times 25$. Since a smaller $k$ requires less computation, we adopt $k=100$ with a $20 \times 20$ filter in subsequent tests.

In order to evaluate how the features $M I, C S S$ and $D$ affect the cup segmentation, we conducted the tests using different combinations of the three types of features. Besides the mean overlapping error $\mu_{E}$, we also compute mean CDR error $\mu_{\delta}$ and AUC of ROC curve in glaucoma detection to evaluate the performance. In addition, we have also compared the proposed method with the threshold method [3], the ASM method [7] and the regression method [6]. Table 2 shows mean overlapping error $\mu_{E}$, the mean CDR error $\mu_{\delta}$ and the AUC of the ROC curves. Fig. 4 shows the ROC curves. Results show that $M I$ works well for glaucomatous subjects while relatively poor for healthy subjects. $C S S$ works better for healthy subjects. $D$ is an important feature that improves the performance significantly. Fig. 5 shows the cup boundaries for five samples by the proposed method and three other methods. From the results, we can see that previous methods can work well when pallor is obvious, but their performance in discs without obvious pallor is less accurate. The proposed method over-estimates very small cups $(\mathrm{CDR}<0.4)$ and under-estimates very large cups $(\mathrm{CDR}>0.8)$ when the pallor is very weak or absent, e.g., the third and last samples. This is because there are very few such very small or very large cups in the data set. When $D$ is included in the feature space where most discs have medium cups, the trained classifier is dominated by the majority medium cups. This limitation is reasonable as these are the most difficult cases even for human eyes. Although the bias exists, the obtained CDRs for very large cups are still high and the CDRs for very small cups are still small, as shown in Fig. 5 . Therefore, we obtain good glaucoma detection results.

\section{Discussions and Conclusions}

In this paper, we present a superpixel classification based cup segmentation for glaucoma detection. It is shown that $M I$ is a good feature as majority of glaucomatous subjects often have relatively obvious pallor. $C S S$ is useful to reducing difficulty due to the uneven illumination. It is less sensitive to the illumination change. The location information further improves the results as it incorporates the prior knowledge of the cup. 
The accuracy of our current method is much better than previous methods [3] 6], 7]. The glaucoma detection accuracy by the proposed method is only slightly below the manual CDR. From the discussions with clinicians, it is good enough for a large-scale glaucoma screening program. However, there are still many aspects for improvement in the proposed methods. For example, the proposed method under-estimates the very large cups while over-estimating the very small cups when pallor is very weak or absent. In addition, CDR based screening also has its limitations. Therefore, combining CDR with other factors are expected to further improve the performance. In the future, we would explore the integration of other factors to improve diagnosis outcomes toward a more reliable and efficient glaucoma screening system.

\section{References}

1. Quigley, H.A., Broman, A.: The number of people with glaucoma worldwide in 2010 and 2020. Br. J. Ophthalmol. 90(3), 262-267 (2006)

2. Damms, T., Dannheim, F.: Sensitivity and specificity of optic disc parameters in chronic glaucoma. Invest. Ophth. Vis. Sci. 34, 2246-2250 (1993)

3. Joshi, G.D., Sivaswamy, J., Karan, K., Krishnadas, R.: Optic disk and cup boundary detection using regional information. In: Proc. IEEE Int. Symp. Biomed. Imag., pp. 948-951 (2010)

4. Joshi, G.D., Sivaswamy, J., Krishnadas, S.R.: Optic disk and cup segmentation from monocular color retinal images for glaucoma assessment. IEEE Trans. on Medical Imaging 30, 1192-1205 (2011)

5. Wong, D.W.K., Liu, J., Lim, J.H., Li, H., Wong, T.Y.: Automated detection of kinks from blood vessels for optic cup segmentation in retinal images. In: Proc. SPIE, vol. 7260, p. 72601J (2009)

6. Xu, Y., Xu, D., Lin, S., Liu, J., Cheng, J., Cheung, C.Y., Aung, T., Wong, T.Y.: Sliding window and regression based cup detection in digital fundus images for glaucoma diagnosis. In: Fichtinger, G., Martel, A., Peters, T. (eds.) MICCAI 2011, Part III. LNCS, vol. 6893, pp. 1-8. Springer, Heidelberg (2011)

7. Yin, F., Liu, J., Wong, D.W.K., Tan, N.M., Cheung, C., Baskaran, M., Aung, T., Wong, T.Y.: Automated segmentation of optic disc and optic cup in fundus images for glaucoma diagnosis. In: IEEE Int. Symp. on Computer-Based Medical Systems, pp. 1-6 (2012)

8. Achanta, R., Shaji, A., Smith, K., Lucchi, A., Fua, P., Susstrunk, S.: Slic superpixels compared to state-of-the-art superpixel methods. IEEE Trans. Pat. Ana. Mach. Intel. 34, 2274-2282 (2012)

9. Itti, L., Koch, C., Niebur, E.: A model of saliency-based visual attention for rapid scene analysis. IEEE Trans. Pattern Anal. Mach. Intell. 20, 1254-1259 (1998)

10. Chang, C.C., Lin, C.J.: LIBSVM: a library for support vector machines (2001), Software available at http://www.csie.ntu.edu.tw/ cjlin/libsvm

11. Fitzgibbon, A., Pilu, M., Fisher, R.B.: Direct least squares fitting of ellipses. IEEE Trans. on Pat. Anal. and Mach. Intel. 21(5), 476-480 (1999)

12. Cheng, J., Liu, J., Xu, Y., Yin, F., Wong, D.W.K., Tan, N.-M., Cheng, C.-Y., Tham, Y.C., Wong, T.Y.: Superpixel classification based optic disc segmentation. In: Lee, K.M., Matsushita, Y., Rehg, J.M., Hu, Z. (eds.) ACCV 2012, Part II. LNCS, vol. 7725, pp. 293-304. Springer, Heidelberg (2013) 